\title{
12. Asylpolitik und Migrationspolitik
}

\section{Rosita Fibbi}

\section{OpenEdition}

\section{Journals}

Electronic version

URL: http://journals.openedition.org/sjep/274

DOI: $10.4000 /$ sjep. 274

ISSN: 1663-9677

\section{Publisher}

Institut de hautes études internationales et du développement

\section{Printed version}

Date of publication: 1 avril 2003

Number of pages: 217-234

ISBN: 2-88247-050-3

ISSN: $1660-5926$

\section{Electronic reference}

Rosita Fibbi, «12. Asylpolitik und Migrationspolitik », Schweizerisches Jahrbuch für Entwicklungspolitik [Online], 22-1 | 2003, Online erschienen am: 06 Mai 2010, abgerufen am 08 September 2020. URL http://journals.openedition.org/sjep/274 ; DOI : https://doi.org/10.4000/sjep.274 


\section{ASYLPOLTTIK UND MIGRATIONSPOLITIK*}

7 WEI TRENDS prägen gegenwärtig die schweizerische Asyl- und Migrationspolitik: Der langwierige Gesetzgebungsprozess kontrastiert mit den raschen Veränderungen bei der Herkunft der Migrantinnen und Migranten.

Bereits zum dritten Mal in Folge wird an dieser Stelle auf die Revisionen des Asylgesetzes, des Ausländergesetzes und des Einbürgerungsgesetzes eingegangen, die sich in mehr oder weniger fortgeschrittenem Stadium befinden. Bei der Suche nach mehrheitsfähigen Konsenslösungen muss stets auf bestimmte politische Akteure Rücksicht genommen werden, die mit immer neuen Volksinitiativen die politische Richtung massgeblich beeinflussen und den Gesetzgebungsprozess verlangsamen. Die bisher verfügbaren Daten der Volkszählung 2000 geben Aufschluss über die Veränderungen bei den Migrationsströmen und der Bevölkerungsstruktur.

\subsection{ASYLPOLITIK}

\section{$\square$ Eingereichte Asylgesuche}

Rund 20’000 Personen haben 2001 in der Schweiz um Asyl angesucht. Dies sind 17,2 Prozent mehr als im Vorjahr. Allerdings war im Jahr 2000 bei der Zahl der eingereichten Asylgesuche einer der niedrigsten Werte der vergangenen zehn Jahre verzeichnet worden. Die Zahl der 2001 eingereichten Gesuche entspricht ungefähr dem jährlichen Mittel der 90er Jahre (Grafik 2).

\section{Grafik 2: Entwicklung der Asylgesuche (1991-2001)}

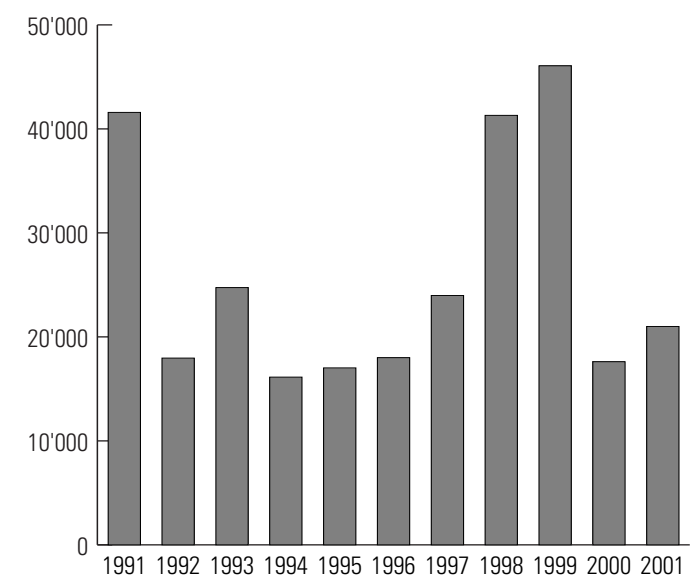

Quelle: Bundesamt für Flüchtlinge, Asylstatistik 2001, BFF, Januar 2002.

giften, die Lähmungen mit meist tödlicher Folge hervorrufen.

* Von Rosita Fibbi, Soziologin, Institut d'anthropologie et de sociologie; Schweizerisches Forum für 
Die zahlenmässig stärksten Gruppen von Asylsuchenden stammen aus der Balkanregion, nämlich aus der Bundesrepublik Jugoslawien (16\%), BosnienHerzegowina (6\%) und Mazedonien (4,3\%), gefolgt von Personen aus der Türkei $(9,5 \%)$ und aus dem Irak $(5,8 \%)$.

Tabelle 26: In der Schweiz eingereichte Asylgesuche 1991-2001 nach Herkunftsland

\begin{tabular}{lrrrrrrrrrr}
\hline Herkunftsland & $\mathbf{1 9 9 2}$ & $\mathbf{1 9 9 3}$ & $\mathbf{1 9 9 4}$ & $\mathbf{1 9 9 5}$ & $\mathbf{1 9 9 6}$ & $\mathbf{1 9 9 7}$ & $\mathbf{1 9 9 8}$ & $\mathbf{1 9 9 9}$ & $\mathbf{2 0 0 0}$ & $\mathbf{2 0 0 1}$ \\
\hline Algerien & & 751 & 303 & 388 & 396 & 564 & 529 & 491 & 477 & 828 \\
\hline Bosnien-Herzegowina & & 6878 & 3343 & 3534 & 1269 & 1987 & 1891 & 1513 & 1304 & 1230 \\
\hline Kongo (Demokr. Rep.) & 677 & 362 & 276 & 320 & 695 & 605 & 536 & 523 & 540 & 602 \\
\hline Guinea & & & & & & 193 & 335 & 388 & 455 & 679 \\
\hline Irak & 117 & 79 & 151 & 321 & 413 & 522 & 2041 & 1658 & 908 & 1201 \\
\hline Mazedonien & & & & & & & & & 64 & 884 \\
\hline BR Jugoslawien & 6262 & 5291 & 4124 & 5491 & 6223 & 6913 & 20396 & 28913 & 3613 & 3425 \\
\hline Sierra Leone & & & & & & 178 & 222 & 756 & 395 & 618 \\
\hline Sri Lanka & 2826 & 1744 & 1487 & 1024 & 1965 & 2137 & 1901 & 1487 & 898 & 684 \\
\hline Türkei & 1827 & 1205 & 1068 & 1293 & 1317 & 1395 & 1565 & 1453 & 1431 & 1960 \\
\hline Andere & 6086 & 8356 & 5194 & 4451 & 5710 & 8696 & 11557 & 8886 & 7526 & 8522 \\
\hline Gesamt & $\mathbf{1 7 9 6 0}$ & $\mathbf{2 4 7 3 9}$ & $\mathbf{1 6 1 3 4}$ & $\mathbf{1 7 0 2 1}$ & $\mathbf{1 8 0 0 1}$ & $\mathbf{2 3 9 8 2}$ & $\mathbf{4 1 3 0 2}$ & $\mathbf{4 6 0 6 8}$ & $\mathbf{1 7 6 1 1}$ & $\mathbf{2 0 6 3 3}$ \\
\hline
\end{tabular}

Quelle: Bundesamt für Flüchtlinge, Asylstatistik 2001, BFF, Januar 2002.

Hinweis: Die vorliegende Tabelle fasst die Daten der jährlichen BFF-Publikation zusammen, in der die wichtigsten Herkunftsländer der Asylsuchenden ausdrücklich erwähnt werden. So erscheinen in der Statistik Länder, die zuvor nicht erfasst worden waren (in diesem Jahr Mazedonien). In der vorliegenden Tabelle sind alle in der Veröffentlichung des BFF genannten Länder aufgeführt, um die Veränderungen bei der Herkunft der Asylsuchenden zu veranschaulichen.

Innerhalb der zahlenmässig stärksten Gruppe von Asylsuchenden aus der Balkanregion (26,9\% der eingereichten Gesuche) haben vor allem die Asylgesuche von aus Mazedonien stammenden Personen gegenüber dem Vorjahr markant von 64 auf 884 zugenommen. Damit befindet sich Mazedonien erstmals unter den zehn bedeutendsten Herkunftsländern der Asylsuchenden.

Ebenfalls zugenommen hat die Zahl der Asylsuchenden aus Afrika südlich der Sahara. Ihr Anteil an den eingereichten Gesuchen hat sich innerhalb von zwei Jahren von 8,9 Prozent (1999) auf 22,5 Prozent (2001) erhöht. Allein in der ersten Jahreshälfte 2002 wurden 6197 Asylgesuche von Afrikanerinnen und Afrikanern eingereicht. Dies sind rund 33 Prozent mehr als im gesamten Jahr 2001. Zum überwiegenden Teil handelt es sich dabei um allein stehende Männer. Diese Entwicklung führte dazu, dass sich die Verfahren in die Länge ziehen, denn die Situation dieser Personen unterscheidet sich stark von jener der Asylsuchenden aus der Balkanregion. Die Feststellung der Identität dieser Personen wird dadurch erschwert, dass ihr Herkunftsland oft keine diplomatische Vertretung in der Schweiz besitzt ${ }^{1}$. Angesichts der hohen Zahl von Afrikanerinnen und Afrikanern in der französischen Schweiz wurde der Schlüssel für die Verteilung der Flüchtlinge auf die Kantone von verschiedenen Kreisen in Frage gestellt ${ }^{2}$.

Migrationsstudien.

„Ausländerpolitik unter Druck“, Interview mit Bundesrätin Ruth Metzler, Tages-Anzeiger, 6.7.2002.

„Souvent non expulsables, peu contrôlables, les requérants issus d'Afrique centrale et de l'Ouest sont majoritairement placés en Suisse romande“, L'Hebdo, 18.7.2002. Die Flüchtlinge werden abhängig von der Wohnbevölkerung auf die einzelnen Kantone aufgeteilt. Der Verfasser des Beitrags weist jedoch darauf hin, dass der Anteil der Asylsuchenden aus Zentral- und Westafrika, gemessen an der Gesamtzahl der Asylsuchenden im entsprechenden Kanton, in keinem einzigen Deutschschweizer Kanton über 19,1 Prozent liegt, während er in der Westschweiz zwischen 21,7 Prozent (Jura) und 31 


\section{$\square$ Befürwortete Asylgesuche}

2001 gewährte das Bundesamt für Flüchtlinge (BFF) 2253 Personen Asyl. Dies sind etwa gleich viel wie im Vorjahr und 9,3 Prozent mehr als 1999. Der Anteil der befürworteten Asylgesuche an allen behandelten Gesuchen belief sich auf 11,7 Prozent. Dieser Wert liegt ziemlich im Zentrum der Bandbreite der vergangenen Jahre (5-15\%).

Rund 8900 Personen wurden 2001 vorläufig aufgenommen (47,4\% weniger als 2000). Dabei handelte es sich primär um Personen aus der Bundesrepublik Jugoslawien und aus Sri Lanka. Zu Beginn des Jahres 2001 kam eine gewisse Anzahl Personen in den Genuss der im Jahr 2000 lancierten „Operation Humanitäre Hilfe“" (siehe JSDW 2001, S. 308). Gesamthaft erhielten mehr als 15'000 Personen im Rahmen dieser Aktion einen regulären Status. Von den vorläufig Aufgenommenen sind 48 Prozent Frauen und 37,5 Prozent Kinder unter 14 Jahren. Der Grund für diese bedeutenden Anteile ist die gestiegene Zahl der Familienzusammenführungen.

Tabelle 27: Personen im Asylbereich (Stand Ende Dezember 2001)

\begin{tabular}{lrrr}
\hline & $\mathbf{3 1 . 1 2 . 2 0 0 0}$ & $\mathbf{3 1 . 1 2 . 2 0 0 1}$ & Veränderung in \% \\
\hline Gesamt & 98480 & 93363 & -5.2 \\
\hline Anerkannte Flüchtlinge & 25534 & 26577 & 4.1 \\
\hline Vorläufige Aufnahme & 32114 & 30734 & -4.3 \\
\hline Vollzug hängig oder blockiert & 13363 & 10398 & -22.2 \\
\hline Erstinstanzlich hängige Gesuche (BFF) & 15137 & 14603 & -3.5 \\
\hline Zweitinstanzlich hängige Gesuche (ARK) & 12332 & 11051 & -10.4 \\
\hline
\end{tabular}

Quelle: Bundesamt für Flüchtlinge, Asylstatistik 2001, BFF, Januar 2002.

Die Verringerung der Zahl der Personen im Asylbereich ist darauf zurückzuführen, dass sich die Zahl der Fälle, die in den Genuss einer Aufnahme aus humanitären Gründen kamen oder deren Fall durch die kantonale Fremdenpolizei geregelt wurde, verdoppelt hat. 11'012 Personen erhielten eine Aufenthaltsbewilligung ${ }^{3}$.

2001 wurden 15'823 Wegzüge aus der Schweiz gezählt. Dies entspricht einem markanten Rückgang gegenüber dem Vorjahr $(-67,7 \%)$, als eine ausserordentlich hohe Zahl von Wegzügen registriert wurde. Während sich im vorangegangenen Jahr die nicht offiziellen Wegzüge dank den Rückkehrhilfeprogrammen reduziert hatten, war ihre Zahl im Berichtsjahr wie gewohnt höher als jene der regulären Wegzüge, der Rückführungen und der Zwangsausschaffungen in einen Drittstaat. $\mathrm{Zu}$ den nicht offiziellen Wegzügen werden Asylsuchende gezählt, die freiwillig, jedoch ohne Meldung zu erstatten, in die Heimat zurückkehren oder in einen Drittstaat auswandern beziehungsweise sich ohne gültige Papiere weiterhin in der Schweiz aufhalten.

1023 Personen, die als anerkannte Flüchtlinge in der Schweiz wohnten, wurde das Asyl entzogen, da sie keinerlei Gefahren mehr ausgesetzt waren. Darüber hinaus entzog das BFF 10'893 Personen die vorläufige Aufenthaltsbewilligung.

Prozent (Neuenburg) beträgt. Die für das Asylwesen zuständigen kantonalen Stellen reichten eine Beschwerde ein, der das BFF grosse Beachtung schenkte. In einer Weisung vom 15. Juli 2001 bekräftigte das Bundesamt, Asylsuchende in Zukunft möglichst gerecht anhand ihrer Nationalität auf die Kantone verteilen zu wollen.

3 Zur Erinnerung: Seit 2000 werden diese Personen nicht mehr zum Asylbereich gerechnet. 
Es handelte sich dabei mehrheitlich um Personen aus Sri Lanka und aus dem ehemaligen Jugoslawien, die im Rahmen der Humanitären Aktion 2000 in den Genuss kantonaler Sonderregelungen gekommen waren.

\section{$\square$ Rückkehr und Rückführung}

Der Vollzug der Rückführungen ist der wohl heikelste Aspekt der gesamten Asylpolitik. Im vergangenen Jahr wurde an dieser Stelle über zwei bedauerliche Fälle aus dem Jahr 2001 berichtet, in denen die Ausschaffungspraktiken zwei Asylsuchenden das Leben kostete (siehe JSDW 2002, S. 234).

Auch der Europäische Ausschuss zur Verhütung von Folter (Comité européen pour la prévention de la torture, CPT) befasste sich mit diesen tödlichen Vorfällen. In seinem am 25. März 2002 in Strassburg erschienenen Bericht über die Behandlung von Personen in schweizerischen Gefängnissen begrüsste der Ausschuss die umfangreichen Bestrebungen zur Vereinheitlichung des schweizerischen Strafverfahrens. Damit wird den wesentlichsten Empfehlungen des CPT zur Verhinderung von Misshandlungen Rechnung getragen. Hingegen übt der CPT scharfe Kritik an den Zwangsrückführungen von Ausländerinnen und Ausländern auf dem Luftweg, da diese ein offensichtliches Risiko für unmenschliche und erniedrigende Behandlung aufweisen. Mit einer Reihe von Grundsätzen will der Ausschuss diese Gefahr eindämmen: Verbot von Zwangsmitteln, die die Atmung behindern können; Ausarbeitung von Vorgehensweisen, um den Erstickungstod zu vermeiden; sachgemässe Schulung des Begleitpersonals ${ }^{4}$.

In seiner Erwiderung wies der Bundesrat nachdrücklich darauf hin, dass die Empfehlungen des CPT bereits heute weitgehend umgesetzt werden und dass derzeit eine gesamtschweizerische Regelung erarbeitet wird. In diesem Zusammenhang ist auch die Schaffung einer neuen Abteilung Vollzugsunterstützung im BFF zu erwähnen. Ihre Aufgabe ist die Beschaffung von Reisepapieren für Personen, die in die Heimat zurückgeführt oder ausgeschafft werden. Dabei arbeitet sie mit den diplomatischen und konsularischen Vertretungen der Heimat- und Herkunftsländer der betroffenen Personen zusammen. Ferner unterstützt sie die Kantone bei der organisatorischen Abwicklung der Rückführungen und Ausschaffungen. Am Flughafen Zürich richtete der Bund eine neue swissREPAT-Dienststelle ein. Im Auftrag der Kantone plant sie die Reisen von Personen, die freiwillig ausreisen wollen oder ausgeschafft werden sollen ${ }^{5}$.

Auch die Schweizerische Akademie der Medizinischen Wissenschaften hat sich mit der Frage der medizinischen Versorgung von inhaftierten Personen befasst und entsprechende Richtlinien ausgearbeitet. Wie auch der Europarat nennt sie gewisse Bedingungen, die von mehreren Haftanstalten in der Schweiz derzeit nicht erfüllt werden, etwa die Notwendigkeit einer hierarchischen Unabhängigkeit des Gesundheitspersonals gegenüber den Vollzugsbehörden ${ }^{6}$. Ein Arzt kann einen Häftling, der sich in einem Zustand grosser Erregung befindet, nicht durch die Verabreichung von Psychopharmaka beruhigen, denn eine solche Massnahme darf nur erfolgen, wenn sie medizinisch notwendig ist und der Patient seine Zustimmung gibt, es sei denn, er ist dazu nicht in der Lage.

4 „Le Comité européen contre la torture épingle la Suisse, qui obéit“, Le Courrier, 26.3.2002; „Des policiers spécialisés devraient bientôt se charger de l'expulsion des réfugiés“", Le Temps, 5.4.2002.

Medienrohstoff des BFF vom 26.6.2002, Leistungsbilanz im Asylbereich.

6 „Contraintes et médecine: des règles pour éviter les dérapages“, Le Temps, 10.1.2002. 


\section{$\square$ Revidiertes Asylgesetz}

Nachdem erst im Oktober 1999 ein umfassend revidiertes Asylgesetz in Kraft getreten ist, zeichnet sich heute bereits eine neue Revision dieses Textes ab. Insgesamt hat sich das Parlament bis heute mehr als hundert Mal mit der Asylfrage befasst, und die Stimmbürgerinnen und Stimmbürger werden an der Urne zu einer neuen Asylinitiative Stellung nehmen müssen. Welche Neuerungen werden mit dieser Gesetzesrevision bezweckt ${ }^{7}$ ?

Die sichere Drittstaatenregelung

Zentrales Element ist zweifellos die so genannte sichere Drittstaatenregelung (siehe JSDW 2002, S. 232). Diese erlaubt es, auf ein in der Schweiz eingereichtes Asylgesuch nicht einzutreten, sofern die betreffende Person aus einem als sicher geltenden Drittstaat eingereist ist, und sie in dieses Land zurückzuführen. Unter der geltenden Gesetzgebung muss sich eine Person während mindestens 28 Tagen in diesem Transitstaat aufgehalten haben, damit die Rückführung in den Drittstaat vollzogen werden kann.

Der Vollzug der Rückführung wird insofern vereinfacht, als der entsprechende Drittstaat vorgängig konsultiert wird und sich zur Rückübernahme der betreffenden Personen bereit erklärt. Die Schweiz hat mit sämtlichen Nachbarstaaten solche gegenseitigen Rückübernahmevereinbarungen getroffen. Auf Grund dieser Bestimmung kann der Bundesrat jene Drittstaaten bezeichnen, die er als sicher erachtet. Dazu dürften namentlich die Nachbarländer der Schweiz zählen. Allerdings werden bei der Anwendung der Drittstaatenregelung einige Sonderregelungen gelten. So kann sie beispielsweise nicht auf Asylsuchende angewendet werden, die nahe Verwandte in der Schweiz haben.

Der Bundesrat befürwortet die Drittstaatenregelung sowohl aus innen- als auch aus europapolitischen Erwägungen. Dank diesem Grundsatz, der an die Initiative ,gegen Asylrechtsmissbrauch“ anknüpft, stellt die Gesetzesrevision in gewissem Sinne einen Gegenvorschlag zur Initiative dar.

Allerdings weist die zuständige Bundesrätin Ruth Metzler jeglichen Vorwurf zurïck, mit der Drittstaatenregelung den Anliegen der Schweizerischen Volkspartei (SVP) Rechnung zu tragen ${ }^{8}$. Im Gegensatz zur SVP-Initiative, die ebenfalls mit dem Begriff des sicheren Drittstaates argumentiert, sollen tatsächlich verfolgte Personen auch künftig in der Schweiz aufgenommen werden, denn die Schweiz wird auf das Asylgesuch eintreten, wenn der Drittstaat die Rückübernahme verweigert oder wenn es sich bei der betreffenden Person offenkundig um einen politischen Flüchtling handelt.

Zweitens entspricht dieser Grundsatz dem Geist des Dubliner Abkommens, wonach abgewiesene Asyl suchende Personen in keinem anderen Mitgliedsstaat des Abkommens aufgenommen werden ${ }^{9}$. Einerseits will die Schweiz verhindern,

„Asylgesetz-Revision nach der Vernehmlassung“, Neue Zürcher Zeitung, 9.10. 2001.

„Zur Asylinsel sollten wir nicht werden“, Interview mit Bundesrätin Ruth Metzler, Tages-Anzeiger, 6.7.2002.

9 Dieses Abkommen, welches die Zuständigkeit für ein innerhalb der Europäischen Union eingereichtes Asylgesuch festlegt, wurde am 15. Juni 1990 in Dublin unterzeichnet. Es bestimmt, welcher Mitgliedsstaat für die Behandlung eines Asylgesuchs zuständig ist, und schliesst damit eine Lücke der Genfer Flüchtlingskonvention. Das Abkommen garantiert Asylsuchenden, dass ihr Asylgesuch von einem Mitgliedsstaat behandelt wird, sofern nicht ein sicherer Drittstaat dafür zuständig ist. Damit 
dass sämtliche Asylsuchenden, die kraft des Dubliner Abkommens abgewiesen worden sind, in der Schweiz ein Gesuch einreichen können, und andererseits will sie über die erforderlichen Mittel verfügen, um abgewiesene Personen in ihr Herkunftsland zurückzuführen. Allerdings weigern sich mehrere - vor allem ostafrikanische - Länder, ihre eigenen Staatsangehörigen aufzunehmen, sofern sie nicht freiwillig in ihre Heimat zurückkehren. Aus diesem Grund soll künftig die Wirtschafts- und Entwicklungshilfe für diese Staaten an den Grundsatz der Rückübernahme gekoppelt werden ${ }^{10}$.

\section{Ein neuer Status : die humanitäre Aufnahme}

Der zweite zentrale Punkt der Revision ist die Neuregelung der vorläufigen Aufnahme. Die heute praktizierte vorläufige Aufnahme soll durch eine humanitäre und eine provisorische Aufnahme abgelöst werden. Damit soll namentlich der Zugang zum Arbeitsmarkt erleichtert werden ${ }^{11}$. Der Sinn dieser Neuregelung erschliesst sich jedoch erst vor dem Hintergrund der jetzigen Lage der Inhaberinnen und Inhaber einer F-Aufenthaltsbewilligung.

Asylsuchende, deren Gesuch zwar abgelehnt wurde, deren Wegweisung jedoch gemäss völkerrechtlichen Bestimmungen auf Grund der Lage im Herkunftsland oder der persönlichen Situation der in der Schweiz lebenden Person nicht zulässig oder nicht zumutbar ist - z.B. aus Krankheitsgründen oder weil die Rückführung nicht vollzogen werden kann, etwa weil das Herkunftsland die Rückübernahme verweigert -, werden per Entscheid des BFF vorläufig aufgenommen und erhalten eine F-Aufenthaltsbewilligung. Personen, deren Gesuch nach mindestens vier Jahren noch immer hängig ist, können per Entscheid der zuständigen Behörden ebenfalls in den Genuss einer vorläufigen Aufnahme kommen. Die Behörden nehmen dabei Rücksicht auf Härtefälle, etwa auf Familien, deren Kinder bereits eingeschult wurden oder beruflich integriert sind.

Die vorläufige Aufnahme kann entzogen werden, wenn sich die Lage im Herkunftsland ändert, so dass der Schutz der Schweiz nicht mehr erforderlich ist, wenn sich der Gesundheitszustand der betreffenden Person verbessert hat oder wenn der Vollzug der Rückführung erneut möglich wird. Allerdings wird die vorläufige Aufnahme nur relativ selten widerrufen, nämlich in rund einem Viertel der Fälle mit F-Aufenthaltsbewilligung. Zwar erfüllen diese Personen die Bedingungen der Genfer Konvention, die für die Erteilung von Asyl erforderlich sind,

wird verhindert, dass Asylsuchende zwischen den Staaten hin- und hergeschoben werden, ohne dass die Zuständigkeit für den Fall eruiert werden kann, und dass eine Person gleichzeitig oder nacheinander mehrere Gesuche stellen kann. Das Abkommen trat für die zwölf ursprünglichen Unterzeichnerstaaten am 1. September 1997 in Kraft. Seit dem 1. Oktober 1997 gilt es auch für Österreich und Schweden und seit dem 1. Januar 1998 für Finnland. 2001 traten Island und Norwegen dem Abkommen bei. Im Dezember 2000 verabschiedete der Rat der Europäischen Union eine Verordnung betreffend die Errichtung des Systems „Eurodac“ für den Vergleich digitaler Fingerabdrücke von Asylsuchenden und gewissen anderen ausländischen Personen. Auf dieser Grundlage kann eine zentrale Datenbank mit Fingerabdrücken von Asylsuchenden und illegalen Einwanderern errichtet werden, die den Vollzug der Bestimmungen des Dubliner Abkommens erleichtert. Im Juli 2002 wurden im Rahmen der Bilateralen Verhandlungen II Gespräche zwischen der Schweiz und der EU aufgenommen. Als Grundlage für den Beitritt der Schweiz zu den Abkommen von Schengen und Dublin dienen die entsprechenden Verträge, die mit Island und Norwegen geschlossen wurden.

10 „Zur Asylinsel sollten wir nicht werden“, Interview mit Bundesrätin Metzler, Tages-Anzeiger, 6.7.2002.

11 „Weitere Optimierungsversuche im Asylgesetz“, Neue Zürcher Zeitung, 5.9.2002. 
mehrheitlich nicht; dennoch suchen sie als so genannte Gewaltflüchtlinge gerechtfertigterweise Schutz in einem anderen Land, um einem allgemeinen Klima der Gewalt in ihrer Heimat - Bürgerkrieg oder ethnische Konflikte - zu entgehen.

Mit der Gesetzesrevision wollen die Behörden mittelfristig den rechtlichen Status von Personen regeln, deren weiterer Verbleib im Land von der Entwicklung der Situation in ihrer Heimat abhängt. Bei den Inhaberinnen und Inhabern einer F-Aufenthaltsbewilligung soll künftig nach den Gründen, die für die Erteilung der Bewilligung ausschlaggebend waren, unterschieden werden. Abgewiesene Asylsuchende, deren Rückführung vorläufig nicht vollzogen werden kann (z.B. weil das Herkunftsland die Rückübernahme verweigert), werden künftig provisorisch aufgenommen. Dieser Status entspricht weitgehend der vorläufigen Aufnahme gemäss der geltenden Regelung und schliesst Massnahmen für eine mittelfristige Integration der betreffenden Personen aus.

Hingegen kommen Personen, deren Rückführung unzulässig oder nicht zumutbar ist, in den Genuss einer „humanitären Aufnahme“ (die im ersten Revisionsentwurf als „Aufnahme im Hinblick auf die Integration“ bezeichnet wurde) ${ }^{12}$. Sie sind anderen ausländischen Personen auf dem Arbeitsmarkt gleichgestellt und erhalten Zugang zu Integrationsmassnahmen. Ferner ist im Rahmen dieses Status unter bestimmten Voraussetzungen eine Familienzusammenführung möglich. In der Botschaft zur Gesetzesrevision ist die Rede von 2500 Personen, die diesen Status erhalten sollen ${ }^{13}$.

\section{Weitere Neuerungen}

Das Flughafenverfahren wird zu einem vollständigen, beschleunigten Asylverfahren ausgebaut. Gemäss Entwurf sind die Behörden befugt, ihren Entscheid direkt den betroffenen Asylsuchenden mitzuteilen (auch wenn diese einen gesetzlichen Vertreter haben). Gegen den Entscheid kann innerhalb von fünf Tagen Einspruch erhoben werden. Wird der Asylfall am Flughafen bearbeitet, so kann die Behörde eine Ausschaffungshaft von maximal 20 Tagen verfügen und damit den Vollzug sicherstellen, auch wenn sich die betreffende Person der Kooperationspflicht nicht widersetzt hat. Diese Massnahme soll verhindern, dass abgewiesene Asylsuchende vor dem Vollzug der Rückführung in die Illegalität abtauchen.

Die Finanzierung des Asylbereichs wird effizienter und für den Bund kostengünstiger gestaltet. Das derzeitige Finanzierungsmodell, bei welchem die Zahlungen abhängig von der Dauer des Aufenthalts einer Asyl suchenden Person festgelegt werden, wird durch Globalpauschalen ersetzt, deren Höhe entsprechend dem Status der betreffenden Person und der Fähigkeit der Kantone, die Sozialhilfeausgaben zu senken (siehe JSDW 2002, S. 233), gestaffelt wird.

12 „Ausländerpolitik unter Druck“, Interview mit Bundesrätin Ruth Metzler, Tages-Anzeiger, 6.7.2002.

13 Gemäss einem ersten Entwurf der gegenwärtigen Asylgesetzrevision sollten Personen, die während mindestens sechs Jahren eine F-Aufenthaltsbewilligung besassen, automatisch eine B-Bewilligung erhalten (siehe JSDW 2002, S. 233). Dieser Vorschlag wurde im Rahmen der Vernehmlassung von sämtlichen Kantonen - mit Ausnahme von Basel-Stadt und Genf - verworfen, da sie einen Anstieg der Fürsorgebeiträge zu ihren Lasten befürchteten (während für die Unterstützung von Inhaberinnen und Inhabern einer F-Bewilligung der Bund zuständig ist). „Les cantons s'opposent à l'octroi d'un permis de séjour aux requérants établis depuis six ans“, Le Temps, 19.12.2001. 
Mehrere Kantone wünschen einen grösseren Handlungsspielraum bei der Verwendung der Transferzahlungen, die sie vom Bund zur Deckung ihrer Ausgaben im Asylwesen erhalten. Jene Kantone, die den Vollzug der Wegzüge und Rückführungen am effizientesten gestalten, werden finanziell belohnt. Durch das Zusammenspiel negativer und positiver Anreize sollen die Behörden ermutigt werden, den Aufenthalt von endgültig abgewiesenen Personen so kurz als möglich zu halten. In gewissen Fällen kann der Bundesrat ein Arbeitsverbot erlassen.

Schliesslich soll mit der Revision eine in den Kantonen seit langem übliche Praxis gesetzlich verankert werden, nämlich das Obligatorium für die Krankenversicherung und die eingeschränkte Arzt- und Versicherungswahl für Asylsuchende.

Trotz der Mobilisierung der Verbände, die sich für die Rechte der Frauen einsetzen, wurden in der vorliegenden Revision Vergewaltigung und sexueller Missbrauch nicht als Verfolgungsgründe anerkannt, die einen Anspruch auf Asyl rechtfertigen ${ }^{14}$.

\section{Die Frage der nichtstaatlichen Verfolgung}

Anlässlich des 50-jährigen Bestehens der Genfer Konvention wurden die Vertreter der 140 Unterzeichnerstaaten von der Schweizer Regierung und dem UNOHochkommissariat für Flüchtlinge zu einem Treffen in Genf eingeladen, um die Umsetzung der Konvention von 1951 und ihres Protokolls von 1967 zu verstärken.

Als Antwort auf zwei parlamentarische Interpellationen betreffend die so genannte Schutztheorie erklärte sich das BFF bereit, die Frage der nichtstaatlichen Verfolgung zu prüfen ${ }^{15}$. Weder die Genfer Flüchtlingskonvention noch das schweizerische Asylgesetz definieren ausdrücklich den Begriff des Verfolgers. Bisher erkennt das BFF einer Person, die um Asyl ersucht hat, die Flüchtlingseigenschaft nur dann $\mathrm{zu}$, wenn sie von staatlichen oder quasi-staatlichen Organen verfolgt wird. Eine Mehrheit der westlichen Industriestaaten (ohne Deutschland) ist jedoch zur Schutztheorie übergegangen, d.h. Asyl wird auch dann gewährt, wenn eine Verfolgung durch private Dritte vorliegt. Die Entwicklung läuft mehr und mehr auf eine Anerkennung auch der nichtstaatlichen Verfolgung hinaus.

Diese Untersuchung über die Schutztheorie ist jedoch nicht in den Entwurf der Asylgesetzrevision eingeflossen. Daraus lässt sich schliessen, dass dieser Begriff derzeit noch ziemlich umstritten ist.

\section{Kritik am Entwurf}

Die Verfechter des Asylrechts ${ }^{16}$ kritisieren die Tatsache, dass die Gesetzgebung systematisch an die extremistischen Forderungen der SVP angepasst wird. Die Behörden träten nicht mehr auf die Gesuche ein und beschränkten sich darauf, Asylsuchende in die Transitstaaten auszuweisen. Bei ihren Entscheiden stützten sie sich je länger je weniger auf die Fluchtgründe und zunehmend auf formelle Aspekte, wie z.B. die Identitätspapiere oder den Fluchtweg. Damit werde die Substanz des Asylrechts ausgehöhlt ${ }^{17}$. Ferner stellen die Verfechter des Asyl-

14 „Un livre noir tire le bilan d'une politique d'asile raciste“, Le Courrier, 27.4.2002.

15 Medienmitteilung vom 12. September 2001, BFF prüft Frage der nichtstaatlichen Verfolgung.

16 Stellungnahme der SFH zum Entwurf über die Teilrevision des Asylgesetzes, Bern, 4.9.2001.

17 „Révision du droit d'asile: une urgence ?“, Le Courrier, 15.2.2002. 
rechts fest, dass das neue Finanzierungssystem die Kantone dazu verleitet, die Aufenthaltsdauer der Asylsuchenden aus Kostengründen zu verkürzen.

Begrüsst wird hingegen die Anpassung der Rechtsstellung für Opfer von Gewalt, die höchstwahrscheinlich in der Schweiz bleiben werden. Die SVP ihrerseits verurteilt diesen Vorschlag, denn damit kämen ,asylunwürdige“ Personen in den Genuss grösserer Vorteile als andere. Ähnliche Stimmen wurden innerhalb der CVP laut.

Angesichts eines als zu restriktiv beurteilten Asylgesetzes und einer Initiative, die ihres Erachtens das Ende des Rechts auf Asyl in der Schweiz herbeiführen würde, haben fünf Verbände in einem Schwarzbuch dargelegt, wie die politischen Instanzen der Schweiz gegen die Flüchtlingskommission verstossen und diese missbrauchen. Den Behörden wird vorgeworfen, sie seien weniger auf den Schutz von Personen als auf deren Wegweisung bedacht, und zeigen auf, dass die Abschreckungsmassnahmen die Attraktivität der Schweiz als Zufluchtsort nicht vermindern, sondern vielmehr die Lebensbedingungen von Personen, die häufig verfolgt werden und in den meisten Fällen Anspruch auf Schutz haben, unannehmbar verschlechtern ${ }^{18}$.

Im Herbst 2002 wurde die öffentliche Debatte jedoch weniger von der Asylgesetzrevision als von der Initiative der SVP beherrscht.

\section{$\square$ Volksinitiative ,gegen Asylrechtsmissbrauch“}

Die Volksinitiative ,gegen Asylrechtsmissbrauch“, die am 24. November 2002 zur Abstimmung gelangte, war 1999 lanciert worden, zu dem Zeitpunkt also, als vor dem Hintergrund des Kosovo-Krieges eine ausserordentlich hohe Zahl von Personen in der Schweiz Zuflucht suchten und die Kosten des Asylwesens rapide anstiegen. Die Bundesbehörden, die mit dem neuen Asylgesetz indirekt einen Gegenvorschlag zur Initiative vorlegten, argumentierten, den wichtigsten Forderungen der Initiative - insbesondere bezüglich der Senkung der Kosten sei bereits Rechnung getragen worden. Die übrigen Forderungen der SVP, so auch die Drittstaatenregelung, dürften sich in der Praxis kaum umsetzen lassen.

Die Initiative zielte auf eine Reform des Asylbereichs auf drei Ebenen ab: das systematische Nichteintreten auf Asylgesuche, Sanktionen gegen Fluggesellschaften mit mangelhaften Kontrollen, und schliesslich eine Senkung der Unterstützungsleistungen, die nach Ansicht der Initianten eine grosse Anziehungskraft auf wirtschaftlich benachteiligte Menschen aus der ganzen Welt ausüben.

Das Hauptargument der Initianten war auch von den Behörden wiederholt vorgebracht worden: Die Attraktivität des Landes müsse vermindert werden. $\mathrm{Zu}$ diesem Zweck schlug die SVP vor, die Fürsorgeausgaben zu senken, indem gewisse Leistungen künftig nur noch durch Sachleistungen erbracht werden sollen. Um auch die Gesundheitskosten so tief wie möglich zu halten, sollten die Kantone befugt werden, Ärzte, Zahnärzte und Spitäler zu bestimmen, bei denen

18 Schwarzbuch. Bilanz der Asylpraxis: Auswirkungen der Asylgesetzrevision von 1998. Bern, 2001. Diese Publikation wurde gemeinsam von fünf NGO herausgegeben, nämlich der Freiplatzaktion für Asylsuchende der Region Basel und Zürich, IGA Solothurn/SOS racisme, augenauf Zürich, Centre social protestant Genève und Solidarité sans frontières. 
sich Asylsuchende behandeln lassen können. Abgewiesene Personen schliesslich sollten nur Zugang zu Notfallbehandlungen haben.

Zentrales Element der Initiative ist jedoch die Forderung, nicht auf Asylgesuche von Personen einzutreten, die aus einem als sicher geltenden Drittstaat in die Schweiz gelangt sind, wo sie ein Gesuch gestellt haben oder hätten stellen können. Die grosse Mehrheit der Asylsuchenden (98\%) gelangen auf dem Landweg in die Schweiz. Dabei durchqueren sie sichere Drittstaaten, welche die Asylsuchenden nicht in ihr Herkunftsland zurückführen können, wenn sie dort Verfolgungen ausgesetzt sind.

Diese Forderung wurde von den Verfechtern des Asylrechts, aber auch von den Behörden scharf kritisiert. Der Direktor des BFF zeigte sich besorgt darüber, dass die Initiative nicht zwischen tatsächlich verfolgten Flüchtlingen und Personen unterscheidet, die aus wirtschaftlichen Gründen in der Schweiz um Asyl ansuchen. Da 98 Prozent der Gesuchstellenden auf dem Landweg aus einem Drittstaat einreisen, dürfe die Schweiz nach dem Willen der Initianten in 98 Prozent aller Fälle nicht auf das Gesuch eintreten, handle es sich nun um verfolgte Personen oder nicht ${ }^{19}$.

Der Begriff des sicheren Drittstaates findet sich auch im Gesetzesentwurf des Bundesrates, jedoch hebt sich dieses Konzept deutlich vom Vorschlag der SVP $\mathrm{ab}$ : In der Tat will die Regierung jene Asylsuchenden aus dem Verfahren ausschliessen, die sich in einem sicheren Drittstaat aufgehalten haben, allerdings unter gewissen Vorbehalten: Die Schweiz wird auf das Asylgesuch eintreten, wenn der Drittstaat die Rückübernahme verweigert oder wenn es sich bei der betreffenden Person offenkundig um einen politischen Flüchtling handelt.

Laut SFH würde die Annahme der SVP-Initiative de facto das Ende des Rechts auf Asyl in der Schweiz bedeuten. Auch verschiedene Hilfswerke (Schweizerisches Arbeiterhilfswerk, Caritas) lehnten die Initiative ab. Abgesehen von der grundsätzlichen Opposition wies die SFH darauf hin, der Vorstoss der SVP sei insofern ineffizient, als er den Schwierigkeiten beim Vollzug der Ausweisung nicht Rechnung trage, denn kein Land würde sich zur Rückübernahme einer ausländischen Person bereit erklären, die über sein Territorium in die Schweiz gelangt ist ${ }^{20}$. Am 24. November 2002 wurde die SVP-Initiative mit einem minimalen Stimmenvorsprung abgelehnt. Es war das knappste Abstimmungsergebnis, das je von einer Volksinitiative in der Schweiz erzielt wurde. Bei 50,1 Prozent Nein- und 49,9 Prozent Ja-Stimmen entschieden schliesslich gerade 3400 Stimmzettel über den Ausgang. Hingegen wurde die Initiative von einer Mehrheit der Kantone angenommen (12,5 gegen 10,5). Wenig überraschend war auch die geografische Verteilung der Abstimmungsergebnisse: Während sich die gesamte Ostschweiz einschliesslich Zürich hinter die Initiative der SVP stellte, wurde sie in den französischsprachigen Kantonen mit über 55\%iger Mehrheit und in den Kantonen Bern, Luzern und Tessin mit 50-55 Prozent der Stimmen abgelehnt. Eine Westschweizer Tageszeitung sprach von einer „triumphalen Schlappe für die SVP“21: Sämtliche Kommentatoren waren sich darin einig,

19 „Un requérant sans statut de réfugié ne constitue pas encore un abus“, interview de J.-D. Gerber, Le Temps, 8.10.2002.

20 SFH, Wider eine zugenagelte Schweiz! Bern, 25.9.2002.

21 „L'échec triomphal de l'UDC“, Le Temps, 25.11.2002. 
dass die politische Bedeutung dieser Niederlage über die Asylpolitik hinausgeht. Die Spätfolgen des Ergebnisses dürften sich im Frühjahr 2003 bemerkbar machen, wenn die Eidgenössischen Räte den Entwurf des revidierten Asylgesetzes debattieren. Die Verfechter des Asylrechts weisen darauf hin, dass es schwierig sein dürfte, eine Vorlage zu bekämpfen, die ihrer Ansicht nach dem im November abgelehnten Vorschlag bedenklich nahe steht ${ }^{22}$.

\subsection{MIGRATIONSPOLITIK}

\section{$\square$ Einwanderer in der Schweiz: Erste Ergebnisse der Volkszählung 2000}

Die ersten Ergebnisse der Volkszählung 2000 stellen das traditionelle Bild der Eidgenossenschaft (vier Landessprachen, zwei wesentliche Konfessionen) in Frage und zeigen eine weitaus komplexere und kulturell vielfältigere Schweiz als noch vor zehn Jahren. Die kulturelle Diversität zeigt sich in einem Anteil der ausländischen Wohnbevölkerung von 20,5 Prozent (gegenüber 18,1\% im Jahr 1990). Die zahlenmässige Entwicklung allein sagt jedoch relativ wenig aus: Während in der Vergangenheit die Personen aus dem benachbarten Ausland und aus Spanien die Mehrheit der ausländischen Bevölkerung stellten, sind es heute in erster Linie Menschen aus Ex-Jugoslawien und - in geringerem Masse - aus Portugal. 10 Prozent der Bevölkerung besitzen eine andere Muttersprache als die Landessprachen (gegenüber 8,9\% im Jahr 1990). Serbisch und Kroatisch, Albanisch, Portugiesisch, Spanisch, Englisch, Türkisch oder Kurdisch (in absteigender Reihenfolge) werden von weitaus mehr Menschen als Muttersprache angegeben als das Rätoromanische, immerhin die vierte Landessprache der Schweiz.

Mit dem Wegzug von Personen aus Italien und Spanien verringert sich der Anteil der römisch-katholischen Bevölkerung, während der Islam und die christlich-orthodoxe Kirche durch den Zuzug von Menschen aus den slawischen Ländern an Bedeutung gewinnen. Innerhalb von zehn Jahren hat sich der Anteil der muslimischen Bevölkerung in der Schweiz von 2,2 Prozent auf 4,5 Prozent erhöht. Diese Gruppe zählt heute 310'000 Personen. Dies ist mehr als ein Zehntel der Bevölkerung mit Schweizer Pass.

Der Anteil der Italienerinnen und Italiener hat sich auf Grund der Wegzüge und Einbürgerungen (von $31 \%$ im Jahr 1990 auf 22\% im Jahr 2000) stark verringert. Nach wie vor jedoch stellen sie die grösste Gruppe der ausländischen Wohnbevölkerung in der Schweiz. Demgegenüber haben sich andere Gemeinschaften markant vergrössert: Einer von vier Ausländern stammt aus dem ehemaligen Jugoslawien. Dank der Volkszählung stehen heute erstmals detaillierte Angaben über die Zusammensetzung der aus dieser Region stammenden Bevölkerungsgruppe zur Verfügung. Die Personen aus der heutigen Bundesrepublik Jugoslawien (Serbien, Vojvodina, Montenegro und Kosovo) stellen heute mit 211'000 Personen die zweitgrösste ausländische Bevölkerungsgruppe. Was die neu entstandenen Staaten der Region betrifft, so leben 56'000 Personen aus Mazedonien, 51'000 Personen aus Bosnien-Herzegowina, 44'000 Kroaten und 3000 Slowenen in der Schweiz. Im internationalen Vergleich ist die Zahl der nicht europäischen Einwanderer nach wie vor gering.

22 „Le timide refus de l'initiative UDC annonce le sursis du droit d'asile“, Le Courrier, 25.11.2002. 
Während langer Zeit handelte es sich bei den Einwanderern hauptsächlich um Männer. Heutige Trends zeigen jedoch einen Anstieg des Frauenanteils : Wurden 1990 bei den Einwanderern noch 139 Männer auf 100 Frauen gezählt, waren es im Jahr 2000 nur noch 115. Diese Entwicklung zeigt, dass die Migration heute mehr Familien betrifft als in der Vergangenheit. Dies trifft insbesondere auf die amerikanischen und osteuropäischen Bevölkerungsgruppen zu. Schliesslich liefert die Volkszählung zum ersten Mal Angaben über die neu eingebürgerten Personen: Rund 500'000 Personen (9\% der Wohnbevölkerung mit Schweizer Pass) haben im Laufe ihres Lebens die Schweizer Staatsbürgerschaft erworben ${ }^{23}$.

\section{$\square$ Zulassungspolitik}

\section{In-Kraft-Treten der bilateralen Verträge}

Am 1. Juni 2002 sind die im Jahr 2000 unterzeichneten bilateralen Verträge zwischen der Schweiz und der Europäischen Union (siehe JSDW 2001, S. 316) in Kraft getreten. Der Vertrag über den freien Personenverkehr regelt die gegenseitige schrittweise Öffnung des Arbeitsmarktes (siehe JSDW 2002, S. 236).

Auf Verwaltungsseite macht dies namentlich eine Reorganisation der Ausländerstatistik erforderlich. Als Ersatz für das heutige zentrale Ausländerregister (BFA) und für das AUPER (BFF) wird ein neues Instrument mit dem vorläufigen Namen „Ausländer 2000“ errichtet. Das neue System soll leistungsfähiger sein - zusätzliche Möglichkeiten zur Datenverarbeitung - und entspricht den geltenden Anforderungen in Bezug auf den Datenschutz. Dank seinem modularen Aufbau dient es nicht nur den zuständigen Bundesämtern als Arbeitsinstrument, sondern wird von der Einreise der ausländischen Person über den Aufenthalt bis zum Verlassen der Schweiz sämtliche Daten umfassen, die für die Beobachtung des Migrationsphänomens erforderlich sind ${ }^{24}$.

Neuer Entwurf für das Ausländergesetz

Zwanzig Jahre nach der Ablehnung eines Entwurfs für ein neues Ausländergesetz durch das Volk (1982) überwies der Bundesrat den Eidgenössischen Räten im Frühjahr 2002 eine neue Vorlage (AuG). In früheren Ausgaben des Jahrbuchs wurde über die Grundzüge des Entwurfs (siehe JSDW 2001, S. 317) sowie über die im Vernehmlassungsverfahren geäusserte Kritik (siehe JSDW 2002, S. 236) bereits berichtet. Der in der Vernehmlassung deutlich gewordene Widerstand gegen das Projekt ist weitgehend abgeflacht, so dass die derzeitige Fassung nur geringfügig vom ursprünglichen Entwurf abweicht.

Der Hintergrund, vor dem die Ausarbeitung des Gesetzesentwurfs stattfand, war von der demografischen Entwicklung und dem Abschluss der bilateralen Verträge geprägt. Die Migration wird nicht mehr nur als Instrument zur Steuerung des Arbeitsmarktes, sondern als wesentlicher Beitrag zur künftigen Entwicklung des Landes betrachtet. In der Botschaft an das Parlament werden die demografischen Herausforderungen ausführlich erläutert ${ }^{25}$ : Die Bevölkerung wächst, wird aber älter, ab dem Jahr 2030 in etwa ist mit einem Rückgang der Bevölkerung

23 Haug, Werner, Martin Schuler, Philippe Wanner, 2002, Räumliche und strukturelle Bevölkerungsdynamik der Schweiz 1990-2002, Neuchâtel, BFS.

24 BFA, Pressemitteilung vom 29.5.2002, Neues Informationssystem im Ausländer- und Asylbereich.

25 Botschaft zum Bundesgesetz über die Ausländerinnen und Ausländer, Bern, 21.5.2002, BB1 Nr. 20, S. 3709-3850. 
und ab 2015 mit einer Abnahme der berufstätigen Wohnbevölkerung zu rechnen (Botschaftstext, S. 3722-3724). Da die Bedingungen für die Einreise und den Aufenthalt von Bürgern der EU- und EFTA-Staaten durch die bilateralen Verträge geregelt sind, wird der Geltungsbereich des neuen Ausländergesetzes auf aus Drittstaaten stammende Personen beschränkt sein. Rund 40 Prozent der heute in der Schweiz lebenden Ausländer sind nicht EU- oder EFTA-Bürger.

Der Gesetzesentwurf bezieht sich im Wesentlichen auf drei Teilbereiche: die Zulassung und Regelung der Anwesenheit, die Integration ausländischer Personen, sowie Sanktionen bei Missbrauch.

Während die Zulassung für EU- und EFTA-Bürger liberaler gestaltet wird, müssen Personen aus Drittstaaten mit einer restriktiveren Praxis rechnen ${ }^{26}$. Bei den Neuzulassungen sind namentlich die kulturellen und wissenschaftlichen Bedürfnisse der Schweiz sowie die demografischen und sozialen Entwicklungen zu berücksichtigen (Art. 16).

Bereits seit 1991 ist der Zugang von Angehörigen aus Drittstaaten zum schweizerischen Arbeitsmarkt qualifizierten Personen vorbehalten: Laut Gesetzesentwurf sollen Bewilligungen zur Ausübung einer Erwerbstätigkeit nur Führungskräften, Spezialisten und anderen qualifizierten Arbeitskräften, anerkannten Personen aus Wissenschaft, Kultur und Sport sowie Personen im Rahmen eines Kadertransfers von international tätigen Unternehmen erteilt werden (Art. 23). Diese Formulierung bietet einen gewissen Auslegungsspielraum: neben regionalen wirtschaftlichen Bedürfnissen können auch Personen mit nicht-universitären Qualifikationen berücksichtigt werden.

Im Hinblick auf die Anforderungen an Anwärter auf eine Aufenthaltsbewilligung werden nicht nur die beruflichen Qualifikationen ausdrücklich erwähnt, sondern auch die Voraussetzungen für eine nachhaltige Integration. Dazu gehören das Alter, die Sprachkenntnisse und die berufliche und soziale Anpassungsfähigkeit. Von diesen Regelungen betroffen sind nach dem jetzigen Stand der Dinge ein Kontingent von 4000 Aufenthalts- und 5000 Kurzaufenthaltsbewilligungen.

Die erwähnten Bestimmungen sollen es ermöglichen, die Einwanderung als wirtschaftspolitisches Instrument zu nutzen. Allerdings sieht der Entwurf gewisse Ausnahmen vor, bei denen der ökonomische Aspekt der Einwanderung in den Hintergrund rückt: Die vorgesehenen Ausnahmen betreffen schwerwiegende persönliche Härtefälle, Opfer von Menschenhandel und den Schutz vor Ausbeutung (insbesondere Frauen, die zur Prostitution gezwungen werden) (Art. 30).

Der zweite zentrale Aspekt des Entwurfs ist die Integration der zugelassenen Einwanderer und die vorsichtige Ausweitung ihrer Rechte. Das Saisonnier-Statut wird formell abgeschafft und durch eine Kurzaufenthaltsbewilligung ersetzt, die grundsätzlich ein Jahr gültig ist und auf zwei Jahre verlängert werden kann. Die wesentlichste Neuerung gegenüber dem Saisonnier-Statut ist der Anspruch auf Familiennachzug, der von Einwandererkreisen seit vierzig Jahren gefordert wird. Hingegen ist die berufliche Mobilität nach wie vor eingeschränkt. Im Gegensatz zur geltenden Regelung gibt es kein System, welches die Umwandlung der Aufenthaltsbewilligung in eine Niederlassungsbewilligung vorsieht.

26 Dieses duale Zulassungssystem wurde im Zuge der Abschaffung des Drei-Kreise-Modells im Jahr 1998 (JSDW 1999, S. 176) eingeführt. 
Darüber hinaus regelt der Entwurf auch den Nachzug der ausländischen Familienangehörigen von Schweizerinnen und Schweizern: Sie haben Anspruch auf eine Aufenthaltsbewilligung - und nach Ablauf von fünf Jahren auf eine Niederlassungsbewilligung -, sind jedoch verpflichtet, mit dem Ehepartner im gemeinsamen Haushalt zu leben. Inhaberinnen und Inhaber einer Aufenthaltsbewilligung haben Anspruch auf Nachzug des Ehegatten und der Kinder unter 18 Jahren $^{27}$. Die Eltern sind verpflichtet, ihre Kinder innerhalb von maximal fünf Jahren nach ihrer Ankunft in der Schweiz nachzuziehen, damit diese die Landessprache so früh wie möglich erlernen.

Damit wird die Integration erstmals - und im Unterschied zur ersten Fassung des Entwurfs ${ }^{28}$ - als Grundsatz und Ziel der Migrationspolitik anerkannt: Das neue Gesetz dürfte die Eingliederung von Ausländerinnen und Ausländern im Vergleich zu früher erleichtern, verlangt von ihnen aber im Gegenzug einen offenkundigen Willen, sich zu integrieren, namentlich durch das Erlernen der Sprache. Der Entwurf bekräftigt, dass das Zusammenleben zwischen Schweizern und Ausländern auf gemeinsamen Grundprinzipien beruhen muss, nämlich auf Rechtsstaatlichkeit, Respekt und gegenseitiger Toleranz. Der Gesetzesentwurf verankert die bisherige Rückkehr- und Rückführungspraxis und sieht für ausländische Personen, die wegen Krieg oder allgemeiner Gewalt ihre Heimat verlassen mussten, eine Rückkehrhilfe vor.

Der dritte Aspekt des Entwurfs ist die Missbrauchsbekämpfung. Während auf der einen Seite die Rechtsstellung der Ausländerinnen und Ausländer in gewisser Hinsicht konsolidiert wird, sieht der Entwurf auf der anderen Seite verstärkte Sanktionen zur Bekämpfung der Schlepperkriminalität, der Schwarzarbeit und der Scheinehen vor. Fluggesellschaften, die Passagieren mit ungenügenden oder gefälschten Identitätspapieren die illegale Einreise in die Schweiz erleichtern, weil sie gegen die Sorgfaltspflicht bei der Kontrolle der Reisepapiere verstossen haben, sollen bestraft werden (so genannte Carrier Sanctions).

Im Vorfeld der parlamentarischen Debatte werden im Wesentlichen zwei Aspekte des Entwurfs kritisiert: Angesichts der ausserordentlich selektiven Zulassungspolitik wird eine Zunahme der Sans-Papiers befürchtet (Personen, die ungeachtet ihrer Ausbildung schlecht qualifizierte Tätigkeiten ausüben und keine Aussicht haben, jemals einen geregelten Status zu erlangen) ${ }^{29}$. Zudem besteht das Risiko, dass die Unterscheidung zwischen EU-Bürgern und Drittstaatsangehörigen zwei Klassen von Ausländern schafft, wodurch die Verwirklichung des Integrationsgedankens zunichte gemacht werden könnte ${ }^{30}$.

27 Inhaber einer Kurzaufenthaltsbewilligung besitzen keinen Anspruch auf Familiennachzug, können aber einen entsprechenden Antrag stellen.

28 „Walter Schmid: Klare Verbesserungen in der Integration“, Tages-Anzeiger, 9.3.2002.

29 Eduard Gnesa, Direktor des Bundesamtes für Ausländerfragen, ist der Ansicht, dass das Potenzial nicht qualifizierter Arbeitskräfte innerhalb der EU noch nicht ausgereizt ist, so dass ein entsprechender Bedarf durch Personen aus der EU gedeckt werden könnte. „Es wird immer illegale Aufenthalter geben“, Der Bund, 9.3.2002.

30 „Walter Schmid: Klare Verbesserungen in der Integration“, Tages-Anzeiger, 9.3.2002; Peter Hug, „Zwei Klassen Ausländer“, Tages-Anzeiger, 9.3.2002; „La politique des deux cercles est taxée de racisme d'Etat", Le Courrier, 12.3.2002. 


\section{$\square$ Sans-Papiers}

Im Rahmen einer Studie des Schweizerischen Forums für Migrationsstudien wurde untersucht, welche Bedeutung Personen aus dem Asylbereich auf dem schweizerischen Arbeitsmarkt haben und unter welchen Umständen sich ihre wirtschaftliche Eingliederung vollzieht ${ }^{31}$. Die Studie zeigt, dass die insgesamt rund 15'000 Asylsuchenden mit einer N- oder F-Aufenthaltsbewilligung sich nur in geringem Masse wirtschaftlich integrieren können, da sie einen zahlenmässig konstanten und konjunkturunabhängigen Pool von Arbeitskräften bilden, die vornehmlich im Hotel- und Gastgewerbe, in der Landwirtschaft und in der Reinigungsbranche beschäftigt werden ${ }^{32}$. Immerhin stiess die Studie auf ein gewisses Echo, denn im zweiten Teil der Untersuchung wird die äusserst heikle Frage nach dem Umfang der Schwarzarbeit, die von ausländischen Personen ohne geregelten Status erbracht wird, erörtert ${ }^{33}$. Dieser Aspekt wird im Entwurf des Ausländergesetzes durch die Bestimmungen zur Bekämpfung der Schwarzarbeit und im Asylgesetz durch die Regelung des Zugangs von Asylsuchenden zum Arbeitsmarkt abgedeckt. Die Spekulationen über den Umfang des Phänomens der Schwarzarbeit erreichten im Herbst 2001 ihren Höhepunkt ${ }^{34}$. Die nach langem endlich veröffentlichte Studie stützt sich auf Aussagen von rund 1300 Arbeitgebern und spricht von zwischen 70'000 und 180'000 Ausländern, die ohne Bewilligung in der Schweizer Wirtschaft beschäftigt sind. Diese vorsichtige Schätzung entspricht in etwa den Zahlen, die bereits früher von Gewerkschaftsseite genannt worden waren.

Unter den Sans-Papiers, die häufig einer nicht gemeldeten Erwerbstätigkeit nachgehen, befinden sich aber auch zahlreiche Kinder. Damit stellt sich die Frage nach deren Einschulung ${ }^{35}$. Eine Umfrage des Schweizerischen Verbands des Personals öffentlicher Dienste (VPOD) zeigt auf, dass die Einschulung von Kindern illegaler Einwanderer lückenhaft ist und dass je nach Kanton grosse Unterschiede bestehen, obwohl laut der Bundesverfassung, der Konvention über die Rechte der Kinder und des Internationalen Paktes über wirtschaftliche, soziale und kulturelle Rechte sämtliche Kinder in der Schweiz Anrecht auf eine Schulbildung haben ${ }^{36}$. Dieser Missstand dürfte mittelfristig gravierende Folgen nach sich ziehen.

\section{Rundschreiben vom Dezember 2001}

Das Rundschreiben „Praxis der Bundesbehörden bei der Anwesenheitsregelung von Ausländerinnen und Ausländern in schwerwiegenden persönlichen Härtefäl-

31 Piguet, Etienne und Losa Stefano, 2001, Travailleurs de l'ombre? Zürich, Seismo.

32 „Arbeit Asylsuchender - begrenzt und wichtig/Vor allem Hilfskräfte in Landwirtschaft und Gastgewerbe“, Neue Zürcher Zeitung, 1.2.2002.

33 Das Phänomen der Schwarzarbeit ist längst nicht nur auf die Ausländer beschränkt. Allerdings sind sie die einzigen, die damit gegen das Gesetz über Aufenthalt und Niederlassung der Ausländer verstossen, denn illegal im Land lebende Ausländer dürfen keiner Erwerbstätigkeit nachgehen. EfionayiMäder, Denise und Cattacin, Sandro, 2001, Illegal in der Schweiz: eine Übersicht zum Wissensstand in Asyl 1/02, SFH, Bern.

34 „Une personne sans permis sur trois occupée dans le tertiaire?“, La Liberté, 8.11.2002; „SansPapiers: „Pas assez scientifique‘ aux yeux de Ruth Metzler, le rapport commandé par l'Office des réfugiés est bloqué. Une autre étude révèle le pot aux roses : banques, assurances et sociétés informatiques emploient des sans-papiers“.

35 „L'impact des migrations clandestines se ressent dans les écoles lausannoises“, Le Temps, 3.4.2002.

36 „Enfants sans statut régulier mal scolarisés. Le droit à l'éducation n’existe pas pour tous en Suisse, malgré la Constitution, Le Courrier, 9.10.2002. 
len“ vom Dezember 2001 stellt eine implizite Antwort der Behörden auf die Frage der Sans-Papiers dar. Gemäss geltendem Gesetz müssen Ausländerinnen und Ausländer, die sich illegal im Land aufhalten, die Schweiz verlassen, und abgewiesene Asylsuchende, die nicht vorläufig aufgenommen worden sind, können keine Aufenthaltsbewilligung erhalten. Das Rundschreiben stellt aus juristischer Sicht insofern eine Neuerung dar, als vorgeschlagen wird, die Regelung für Härtefälle im Asylbereich auf illegal anwesende Personen auszudehnen ${ }^{37}$.

Bereits im Rahmen der „Humanitären Aktion 2000“ hatte der Bundesrat rund 12'000 Personen ohne geregelten Status die vorläufige Aufnahme gewährt. Dabei orientierte er sich am Asylgesetz, welches diese Möglichkeit für Personen vorsieht, deren Verfahren nach vier Jahren noch nicht abgeschlossen ist. Bei der Hälfte dieser Personen handelte es sich um abgewiesene Asylsuchende. Mit ihrem Rundschreiben vom Dezember 2001 weitete die Regierung den Geltungsbereich dieser Bestimmung auf illegal Anwesende aus.

Ausschlaggebend für die Erteilung einer B-Aufenthaltsbewilligung im Rahmen dieses Dispositivs sind die Dauer des Aufenthalts, der Grad der Integration, der Schulbesuch der Kinder und der Gesundheitszustand. Allerdings rechtfertigt die Aufenthaltsdauer allein keinen Anspruch auf eine Bewilligung. Bei der Anwendung der Regelung zeigt sich, dass die Dauer von vier Jahren ein Mindestwert ist. In der Tat stellt nur eine Aufenthaltsdauer von zehn Jahren ein ausreichendes Motiv für die Gewährung der Bewilligung dar. Um als Härtefall zu gelten, muss die betreffende Person im Land verwurzelt sein und keine Verbindungen zum Herkunftsland mehr besitzen. Die Tatsache, dass die Person in ihrer Heimat verfolgt wird, ist dabei nebensächlich. Laut dem Rundschreiben werden Personen, die sich eines leichten Vergehens schuldig gemacht haben, von der Regelung nicht ausgeschlossen. Als Härtefälle gelten auch Personen mit einer schweren Krankheit, die im Herkunftsland nicht sachgemäss behandelt werden kann ${ }^{38}$.

\section{Regularisierung in kleinen Schritten}

2001 erreichte die Bewegung der Sans-Papiers ihren Höhepunkt. Die Mobilisierung bewirkte eine Stärkung des Bewusstseins für die Problematik, allerdings kamen nur einige hundert Personen in den Genuss einer Regularisierung ihres Status $^{39}$. Das gemeinsame Rundschreiben von BFF und BFA ist ein typisches Produkt des Föderalismus: Die Bundesbehörden erteilen ihre Genehmigung nur in jenen Fällen, in denen der Wohnsitzkanton grünes Licht gegeben hat. Bis heute konnten auf diese Weise nur gerade 250 Härtefälle abschliessend behandelt werden. Diese geringe Zahl lässt sich dadurch erklären, dass die betreffenden Personen ihre Anonymität aufgeben und sich bei der Fremdenpolizei melden müssen ${ }^{40}$.

Während sich gewisse Kreise im Anschluss an die Veröffentlichung des Rundschreibens eine Lockerung der geltenden Bestimmungen erhofften, machte sich bei anderen rasch Enttäuschung breit: Das Rundschreiben beschränke sich da-

37 Spirig, Werner, „Aufenthaltsrecht aus humanitären Gründen. Die Härtefallregelung als Ausweg im Rechtsstaat“, Neue Zürcher Zeitung, 8.8.2002.

38 „Anerkennung von Härtefällen bei Sans-Papiers. Kriterien der Bundesämter publiziert“, Neue Zürcher Zeitung, 17.1.2002.

39 „Neue Initiative zugunsten der Sans-Papiers“, Neue Zürcher Zeitung, 30.6.2002.

40 „Aufenthaltsrecht aus humanitären Gründen“, Neue Zürcher Zeitung, 8.8.2002. 
rauf, die geltenden Bestimmungen zu zitieren und den Auslegungsspielraum abzustecken, ohne eine Lockerung der Gesetzgebung in Betracht zu ziehen. Die Sans-Papiers, die einen Status irgendwo zwischen ausländischen Arbeitskräften und Asylsuchenden einnehmen, würden auch in Zukunft zwei vollkommen voneinander unabhängigen Gesetzen unterstehen, für deren Vollzug zwei verschiedene Ämter zuständig sind ${ }^{41}$.

\section{$\square$ Integrationspolitik: Einbürgerungsgesetz und politische Rechte}

Der Bundesrat hat einen ambitiösen Entwurf für eine neue Bürgerrechtsregelung vorgelegt (siehe JSDW 2002, S. 240). Diese umfasst zum einen eine Verfassungsänderung und zum anderen eine Revision des Einbürgerungsgesetzes, welche auf die erleichterte Einbürgerung von jungen Ausländern der zweiten Generation abzielt. Dieser Aspekt der Gesetzgebung wurde von der Frage der erleichterten Einbürgerung von Ausländern der dritten Generation getrennt, durch welche das Ius Soli in das schweizerische Recht eingeführt werden soll (wenn auch in abgeschwächter Form, da im Gegensatz zu den USA beispielsweise verschiedene Voraussetzungen erfüllt sein müssen).

Im September 2002 befasste sich der Nationalrat mit dieser Frage und genehmigte den Vorschlag, die für das Ansuchen um Einbürgerung erforderliche Aufenthaltsdauer von zwölf auf acht Jahre zu senken und jungen Ausländerinnen und Ausländern, die in der Schweiz geboren wurden oder ihre obligatorische Schulzeit in der Schweiz absolviert haben, ein erleichtertes Einbürgerungsverfahren zu gewähren. Ferner unterstützte der Nationalrat den Vorstoss, ein Beschwerderecht gegen willkürliche Entscheide zu schaffen, welches allerdings keinen Anspruch auf die Schweizer Staatsbürgerschaft darstellt.

Die Vorlage betreffend die automatische Einbürgerung von Ausländerinnen und Ausländern der dritten Generation (Ausländerkinder, bei denen zumindest ein Elternteil in der Schweiz geboren wurde oder hier den grössten Teil der obligatorischen Schulzeit absolviert hat, Ius Soli) hingegen sorgte für heftigere Debatten. Dabei stehen sich zwei Lager gegenüber: Das eine Lager forderte, dass die Jugendlichen oder ihre Eltern die Einbürgerung ausdrücklich beantragen müssen, während das andere Lager für die automatische Zuerkennung der Schweizer Staatsbürgerschaft bei der Geburt eintrat (wodurch die ursprüngliche Nationalität beibehalten werden kann). Schliesslich führte die Nationalratspräsidentin mit ihrer Stimme einen Stichentscheid herbei. Die Grosse Kammer legte damit eine gewisse Zurückhaltung, wenn nicht gar Zaghaftigkeit an den Tag, die in scharfem Kontrast zum Ehrgeiz des Bundesrates steht, dessen Entwurf dem Zeitgeist und den Herausforderungen, mit denen sich die multikulturelle Gesellschaft eines fortschrittlichen Landes konfrontiert sieht, besser gerecht wird.

Die Teilnahme der ausländischen Bevölkerung am politischen Leben lässt sich neben der Einbürgerung auch durch die Verleihung des aktiven und passiven Wahlrechts fördern. Da diese beiden Optionen häufig als Alternativen wahrgenommen werden, gehen Fortschritte in der einen Frage häufig mit einem Stillstand in der anderen einher. Im Jahr 2002 hingegen konnten auf beiden Gebieten erfreuliche Fortschritte verbucht werden.

${ }^{41}$ „Berne prône une transparence pas encore très limpide“, Le Courrier, 22.1.2002. 
Der Kanton Waadt folgte dem jurassischen Beispiel und schlug vor, Ausländerinnen und Ausländern das aktive und passive Wahlrecht in Gemeinde und Kanton zu verleihen (siehe JSDW 2002, S. 240). Die ursprüngliche Vorlage stiess jedoch in der Vernehmlassung auf resolute Ablehnung. Der Vorschlag, der schliesslich dem Volk unterbreitet wurde, beschränkte sich auf die Gewährung dieser Rechte auf Gemeindeebene und wurde im Rahmen der Abstimmung über die neue Waadtländer Kantonsverfassung im September 2002 genehmigt. Angesichts des hohen Anteils der ausländischen Bevölkerung im Kanton Waadt wurde damit ein beachtlicher Erfolg erzielt. Damit ist die Waadt nach Neuenburg und Jura der dritte Westschweizer Kanton, der Ausländerinnen und Ausländern auf Grund ihres langjährigen Aufenthalts im Land die politischen Rechte zugesteht $^{42}$. Diese offene Haltung der Waadtländer Behörden zeigt sich auch in der Asylpolitik, die für sich die Attribute „menschlich“ und „realistisch“ in Anspruch nimmt und sich durch eine gewisse Distanzierung von den Weisungen aus Bern charakterisiert. Sie fand vor kurzem einen umfassenden Rückhalt im Kantonsparlament ${ }^{43}$.

\section{QUELLEN}

Asyl, Schweizerische Zeitschrift für Asylrecht und Praxis, Schweizerische Flüchtlingshilfe, Bern, Jahrgänge 2000 und 2001.

Asylon, Revue de l'Office fédéral des réfugiés, Jahrgänge 2000 und 2001.

Bundesamt für Flüchtlinge, Pressemitteilungen 2000-2001.

Bundesamt für Flüchtlinge, Asylstatistik 2000, BFF, Januar 2001.

Chronologie der Flüchtlingspolitik, Schweizerische Flüchtlingshilfe, Jahrgänge 2000 und 2001.

Planète exil, Revue de l'Organisation suisse d'aide aux réfugiés, Jahrgänge 2000 und 2001.

Vivre ensemble, Bulletin de liaison pour la défense du droit d'asile, Genève.

Bulletin augenauf, Bern.

Carrefour, Journal des Centres de contact Suisses-immigrés.

\section{INTERNET-ADRESSEN}

UNO-Flüchtlingshochkommissariat (UNHCR) : <www.unhcr.ch>.

Human Rights Watch: <www.hrw.org >.

European Council on Refugees and Exiles (ECRE) (Dachorganisation europäischer Nichtregierungsorganisationen im Asyl- und Flüchtlingsbereich): <www.ecre.org $>$.

Bundesverwaltung: <www.admin.ch/>.

Bundesamt für Flüchtlinge (BFF) : <www.asyl.admin.ch $>$; $<w w w . o d r . a d m i n . c h>$.

Bundesamt für Ausländerfragen (BFA) : <www.auslaender.ch/BfA $>$.

Schweizerische Flüchtlingshilfe(SFH) : <www.sfh-osar.ch $>$.

Komitee gegen die Aushöhlung des Asylrechts : <www.sosf.ch $>$.

Institut für Politikwissenschaft der Universität Bern : <www.anneepolitique.ch $>$.

Association romande contre le racisme: <www.AcorSosRacisme.org >

Le Courrier: <www.lecourrier.ch>.

42 Die einzige Gemeinde ausserhalb der französischsprachigen Schweiz, in der Ausländerinnen und Ausländer, die seit mehr als zehn Jahren in der Schweiz niedergelassen sind, das aktive und passive Wahlrecht besitzen, ist unseres Wissens Wald im Kanton Appenzell-Ausserrhoden (seit Dezember 1999) (JSDW 2001, S. 319).

43 „Les largesses vaudoises sur l'asile passent bien“, Le Courrier, 9.10.2002. 